\title{
ANALYSIS OF THE STOCHASTIC TECHNICAL STABILITY OF ENGINEERING STRUCTURES ON AN EXAMPLE OF A MOVING CAR
}

\author{
JERZY KISILOWSKI \\ Kazimierz Pulaski University of Technology and Humanities in Radom, Faculty of Transport and Electrical Engineering, \\ Radom, Poland; e-mail: jkisilow@kisilowscy.waw.pl
}

JAROSŁAW ZALEWSKI

Warsaw University of Technology, Faculty of of Administration and Social Sciences, Warszawa, Poland e-mail: j.zalewski@ans.pw.edu.pl

\begin{abstract}
The article presents analysis of the stochastic technical stability of mathematical models (described by the second-order ordinary differential equations) of technical objects, on the example of a moving motor vehicle with the disturbed location of the center of mass, as a result of a non-uniform load. After conducting simulation of motion of a sport vehicle mathematical model, using the MSC Adams/Car software, executing a maneuver of double lane change without completely returning to the original road lane, interpretation of the results in accordance with the given definition has been attempted. Specifically, the aspect of determining the probability of the solution remaining in a certain area of feasible solutions has been highlighted, as well as determination of the magnitude of this probability for stable motion. In addition, attention has been paid to the selection of the upper limit of probability, for which, under certain conditions, stable motion can be determined.
\end{abstract}

Keywords: stochastic technical stability, mathematical model of motor vehicle, nonlinearity

\section{Introduction}

Analyses on the safety and dynamics of the means of transport seem an important issue from the scientific research point of view. These studies can be performed on real objects or by using their mathematical models and simulating their motion. One of the problems strictly related to the road traffic safety is the stability of the motor vehicle in different traffic conditions, for example with various vehicle load and road surface conditions. For examination of stability various criteria may be used (Börner and Iserman, 2005; Heinzl et al., 2002; Kaneko et al., 2002; Lenasi et al., 1998; Mokhiamar and Abe, 2002), however not all give clear solutions or allow taking into account random disturbances acting on the examined model.

In the presented analysis, definition of the stochastic technical stability (Bogusz, 1972) is used, because of the possibility to consider random external disturbances acting on the system (derived from an uneven road surface for motor vehicles, see Kisilowski and Zalewski (2008a,b), or from the inequalities of the track for railway vehicles, see Kisilowski et al. (1985), Kisilowski and Kardas-Cinal (1993). Furthermore, it is possible to analyse the obtained results on the basis of motion trajectories, which in turn allows making reference to the results or assumptions concerning research on the real motor vehicles.

The aim of this paper is to present examination of the stochastic technical stability on the example of a moving motor vehicle. 


\section{Description of the considered example}

When examining the stochastic technical stability, the following assumptions are made:

- the vehicle body is assumed to be rigid;

- randomly occurring road surface unevenness is described as a stochastic process which is stationary in a broader sense and globally ergodic;

- all coordinate systems are adopted as in Zalewski (2011);

- flexible elements connecting wheel masses to the vehicle body are described by non-linear functions.

Figure 1 shows the physical model of the simulated two-seater sport vehicle in the MSC Adams/Car environment, with degrees of freedom accepted as in Nabagło (2006). This model is assumed to reflect the real vehicle, however no identification concerning this assumption has been performed. The load is represented by two masses corresponding to the driver and the passenger as well as the baggage located in the front of the vehicle (under the bonnet). The location of the center of mass of the vehicle body has been determined in relation to the so-called point "origo" (Fig. 2), which represents the origin of the coordinate system $O_{d} x_{d} y_{d} z_{d}$ located on the road surface, but moving along with the vehicle.

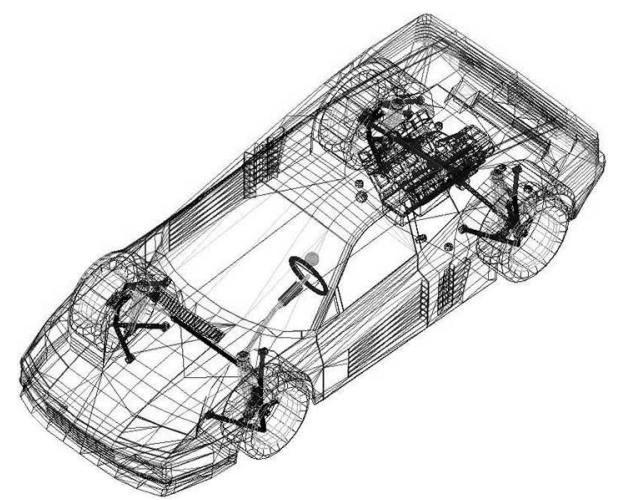

Fig. 1. Full physical model of the examined vehicle. Source: MSC Adams/Car

The suspension in the physical model consists of wishbone arms and McPherson struts. Elastic-damping elements have non-linear characteristics. Power is transferred to the rear wheels, the engine is also mounted at the back of the vehicle. A typical steering system is used.

Assuming that the vehicle body can be represented by a set of cuboids mapping specific parts in a vehicle body, mathematical model of the vehicle is described basing on the works by Gillespie (1992) and Ng (2008), using the equations for translational and rotational motion. It is also supplemented by equations describing both the suspension and the unsprung masses (Nabagło, 2006).

The basic parameters of the sport vehicle model in MSC Adams/Car are:

- mass of the unladen vehicle body $m_{V B}=995 \mathrm{~kg}$;

- nominal coordinates of the center of mass of the unladen vehicle body relative to the "origo": $x_{c}=1.5 \mathrm{~m}, y_{c}=0, z_{c}=0.45 \mathrm{~m}$;

- nominal values of the moments of inertia relative to the axes passing through the "origo" in the unladen vehicle body: $I_{x x}=401.485 \mathrm{~kg} \mathrm{~m}^{2}, I_{y y}=2940.237 \mathrm{~kg} \mathrm{~m}^{2}$, $I_{z z}=2838,750 \mathrm{~kg} \mathrm{~m}^{2}$;

- nominal values of the moments of deviation relative to the axes passing through the "origo" in the unladen vehicle body: $I_{x y}=0 \mathrm{~kg} \mathrm{~m}^{2}, I_{x z}=671.625 \mathrm{~kg} \mathrm{~m}^{2}, I_{y z}=0 \mathrm{~kg} \mathrm{~m}^{2}$. 
For the analysis of motion analysis in extreme conditions, the location of the center of mass in the vehicle body is assumed to have been disturbed resulting from the uneven load. By adding masses of the driver, passenger and baggage, the mass of the entire vehicle is increased, and its distribution in the vehicle changed. The following configuration of disturbances is assumed:

- the vehicle body is loaded with the masses representing the driver $m_{d}=74 \mathrm{~kg}$, passenger $m_{p}=105 \mathrm{~kg}$ and the baggage $m_{b}=45 \mathrm{~kg}$. Their distribution in the vehicle body is shown in Figs. 2 and 3.

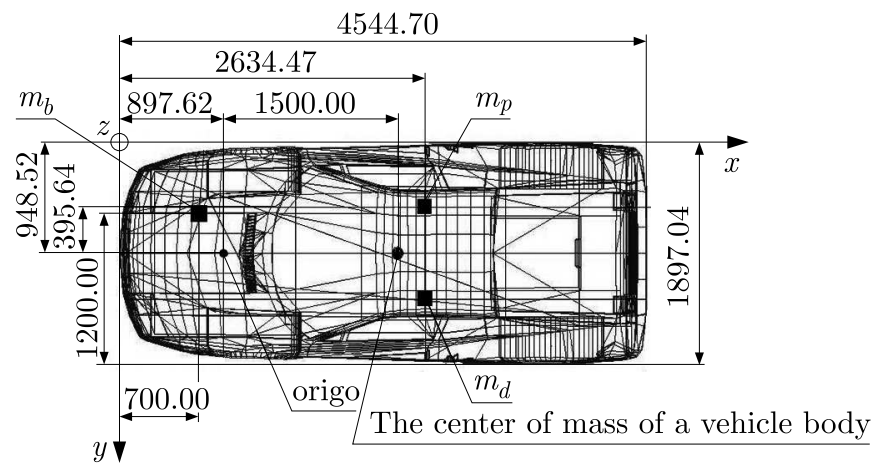

Fig. 2. Distribution of masses loading the vehicle body. Plan view. Source: own research

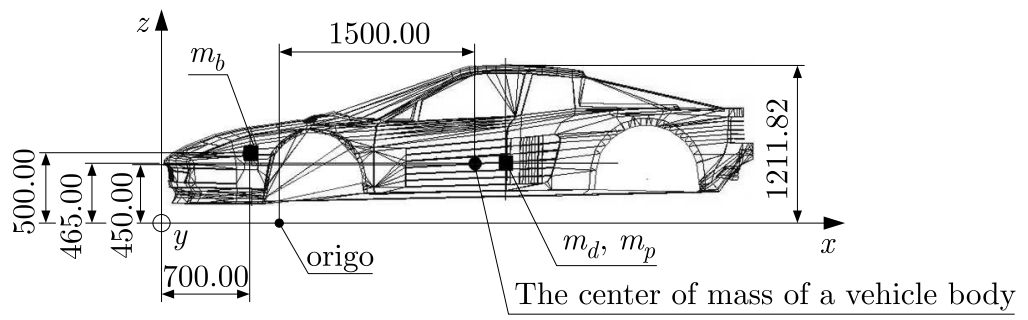

Fig. 3. Distribution of masses loading the vehicle body. Side view. Source: own research

Based on the above assumptions, new coordinates of the center of mass in the laden vehicle body have been determined, which as an effect gave the following results:

- the total mass of the laden vehicle body $m_{V B l}=1174 \mathrm{~kg}$;

- coordinates of the center of mass of the laden vehicle body relative to the "origo": $x_{c}=1.562 \mathrm{~m}, y_{c}=0.016 \mathrm{~m}, z_{c}=0.471 \mathrm{~m}$;

- moments of inertia of the laden body relative to the axes passing through the "origo": $I_{x}=460 \mathrm{~kg} \mathrm{~m}^{2}, I_{y}=3624 \mathrm{~kg} \mathrm{~m}^{2}, I_{z}=3464 \mathrm{~kg} \mathrm{~m}^{2}$;

- moments of deviation of the laden vehicle body relative to the axes passing through the "origo": $I_{x y}=29 \mathrm{~kg} \mathrm{~m}^{2}, I_{x z}=863 \mathrm{~kg} \mathrm{~m}^{2}, I_{y z}=8.8 \mathrm{~kg} \mathrm{~m}^{2}$.

Random disturbances stemming from the road unevenness have been provided using the "2d_stochastic_uneven.rdf" file available in MSC Adams/Car database. The file defines the desired road profile using ARC901 function [16].

Generation of the random disturbances, on the basis of [16], is realised as follows:

- white noise signals are generated based on random variables with almost uniform distribution. Two of these variables are assigned to the road at a distance of every $10 \mathrm{~mm}$;

- the resulting values are integrated over the length of the road argument using the timediscrete filter, in which the independent variable is the road. The effect of this filter is to obtain two approximated realisations of the white noise velocity; 
- the obtained signals, having such properties, become road profiles. According to the definition accepted by Múčka (2009), it is assumed that road waviness for the considered example is 2. The research showed that the waviness obtained for the measured road spectral densities ranges between 1.8 and 2.2, see Múčka (2009);

- after that, both the realisations $z_{1}(s), z_{2}(s)$ are correlated in order to obtain a profile of the road for the left and right wheels $z_{l}(s), z_{r}(s)$ [16]. The correlation coefficient is 0 for the two different road profiles and 1 for the uniform profile. A method of calculating this coefficient is presented the following by formula

$$
\begin{aligned}
& z_{l}(s)=z_{1}(s)+\frac{\operatorname{corr}_{r l}}{2}\left[z_{2}(s)-z_{1}(s)\right] \\
& z_{r}(s)=z_{2}(s)+\frac{\operatorname{corr}_{r l}}{2}\left[z_{2}(s)-z_{1}(s)\right]
\end{aligned}
$$

where $\operatorname{corr}_{r l}$ is the correlation coefficient between the road profiles for the signal realisations $z_{1}(s), z_{2}(s)$.

In the examined vehicle model, the default tire model PAC89 has been removed because it is unable to cooperate with the road profile having random irregularities whose wavelength is smaller than the wheel radius. Instead, the FTRIE model (Flexible Structure Tire odel) has been used, which consists of deformable panels connected by spring elements (Fig. 4).

(a)

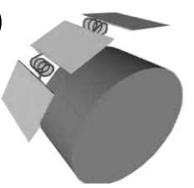

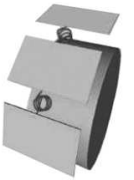

(b)

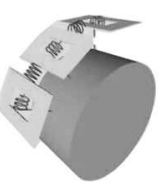

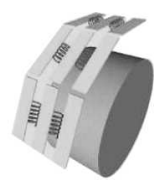

Fig. 4. Schematics of the FTIRE model for (a) radial and (b) circumferential direction. Source: www.cosin.eu

\section{Application of the stochastic technical stability}

The definition of the stochastic technical stability is presented, among others, in Bogusz (1972).

Given a system of stochastic equations:

$$
\frac{d x}{d t}=f(x, t, \xi(t))
$$

where $x=\left(x_{1}, \ldots, x_{n}\right)$ and $f(x, t, y)=\left(f_{1}, \ldots, f_{n}\right)$ and $\xi(t)=\left(\xi_{1}, \ldots, \xi_{n}\right)$ are vectors, whereas $\xi(t)$ for $t \geqslant 0$ is a stochastic process describing randomly occurring disturbances. For the function $f(x, t, y)$, it is assumed that this function is determined for each $x \in E_{n}, y \in E_{n}$ and $t \geqslant 0$. It is also assumed that for the stochastic process $f(0, t, \xi(t))$, there occurs

$$
P\left\{\int_{0}^{T}|f(0, t, \xi(t))| d t<\infty\right\}=1 \quad \text { for each } \quad T>0
$$

Also, the existence of a stochastic process $f(X, t, \xi(t))$ fulfilling the Lipschitz criterion in the interval $[0, T]$ has been assumed, for another process $\eta(t)$ absolutely integrable in the given interval, which can be described as follows

$$
\left|f\left(x_{2}, t, \xi(t)\right)-f\left(x_{1}, t, \xi(t)\right)\right| \leqslant \eta(t)\left|x_{2}-x_{1}\right|
$$


The result of the above assumptions is the existence of only one solution with the initial conditions $t=t_{0}$ and $x\left(t_{0}\right)=x_{0}$, which is an absolutely continuous stochastic process with the probability 1 for $t \geqslant t_{0}$.

It has been as well assumed that in the Euclidean space $E_{n}$ there exist two areas: $\omega$ - limited and open as well as $\Omega$ - limited and closed, where $\omega \subset \Omega$. The existence of a positive number $\varepsilon$, where $0<\varepsilon<1$, and a stochastic process $X(t)$ specified for $t \geqslant t_{0}$ has also been assumed.

The initial conditions of each solution are described as $t=t_{0}, x\left(t_{0}\right)=x_{0}$, and the solution itself as $\left(t, t_{0}, x_{0}\right)$.

The definition of stochastic technical stability is: if every solution to equation (3.1), having its initial conditions in $\omega$, belongs to the area $\Omega$ with the probability $1-\varepsilon$, then system (3.1) is stochastically technically stable towards $\omega$ and $\Omega$ as well as the process $\xi(t)$ with the probability $1-\varepsilon$ (Fig. 5).

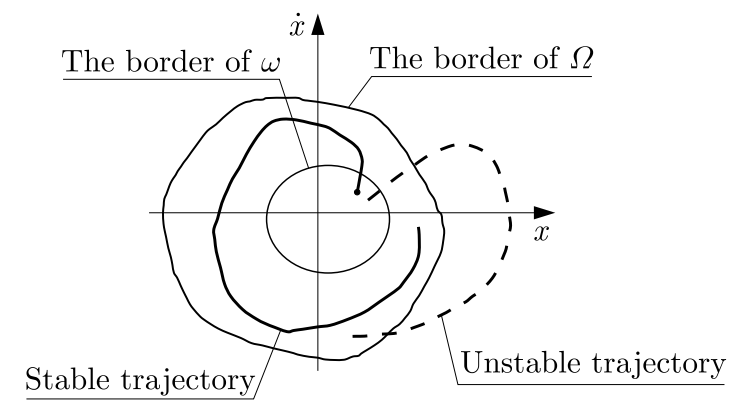

Fig. 5. Graphic interpretation of the stochastic technical stability. Source: Bogusz (1972)

The definition is described by the formula

$$
P\left\{\left(t, t_{0}, x_{0}\right) \in \Omega\right\}>1-\varepsilon \quad \text { for } \quad \bar{x}_{0} \in \omega
$$

To properly apply the given definition, several additional conditions must be fulfilled. At first, it seems necessary to avoid bifurcation, i.e. a double solution to the nonlinear system taken under consideration. In the theory of nonlinear problems, certain cases can be found where there are two or more solutions of the same system, which gives a misleading picture under certain conditions. In this situation, it is necessary to prove the derivativeness and integrability, especially for functions describing randomly occurring disturbances. The integrability of the stochastic process described by condition (3.2) provides an opportunity to analyse the obtained trajectory of the vehicle in terms of stability studies based on the trajectories, while Lipschitz criterion (3.3) ensures the existence of the derivative of the function describing randomly occurring disturbances, originating from the road in this case.

The Lipschitz criterion may be related to the issues presented by Kuratowski (2012), concerning the existence of derivatives of functions. It is important to determine how the stochastic processes $X$ and $\eta$ can be related to variables marked for the curve in Fig. 6. Since in Fig. 6 there are variables $x$ and $x^{\prime}$ that have specific values, so for the processes $X$ and $\eta$ it can also be assumed that at certain moments they adopt the specific values. Thus, as a result, condition (3.3) provides the relevant slope of the process $X$ realisation with respect to the process $\eta$, which is a sort of preservation or warranty of the existence of the derivative in the range between $x_{1}$ and $x_{2}$.

In addition, it is important that the stochastic process describing the road surface irregularities is:

- stationary in a broader sense (time invariant), because as it is known the road does not change in time and the irregularities occurring on its surface are located randomly fixed for a specific, also a stationary reference system; 


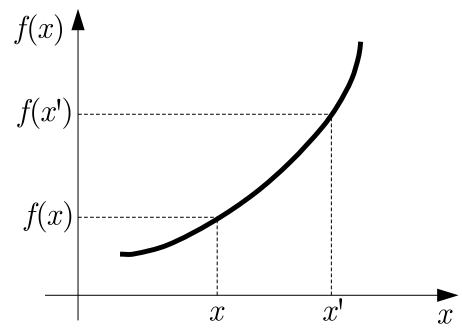

Fig. 6. Graphic interpretation of the Lipschitz criterion for the function $f(x)$. Source: Kuratowski (2012)

- globally ergodic consisting in the fact that the value of the average height of irregularities and their correlation function is equal for various realisations of the process, which provides the ability to conduct analyses based on one realisation. This realisation represents then the whole stochastic process describing road unevenness.

Without the two features mentioned above, it would be impossible to conduct the analysis of the vehicle model motion on the basis of one realisation of the stochastic process describing the randomly occurring road unevenness. It is important from the point of view of disturbances coming from the road. The analysed vehicle model has, as previously mentioned, nonlinear characteristics of its suspension, which still makes it impossible to generalize the obtained results for a set of models with similar features. However, as for the road, providing the above mentioned conditions allows the analysis of various vehicle models on the road with the same characteristics of unevenness.

Another important issue, from the point of view of nonlinear systems analysis, is to determine the initial conditions (the area $\omega$, longitudinal velocity, etc.) as well as a set of feasible solutions $(\Omega)$ in which the solution trajectory is considered to be stable.

The last condition is to determine the significance of the number $\varepsilon$, which defines the range of probability at which the solution remains in the feasible area. This number may be a determinant of the stability of the entire system. It is worth noting that, in accordance with the principle of the center of mass in quasi stiff bodies, the motion analysed is that of a representative point (often the center of mass) to which the entire system is referred.

\section{Analysis and interpretation of the simulation results}

An incomplete double lane change maneuver is analysed, where at the end of the maneuver the vehicle remains roughly in the middle of the width of a single-lane road. The simulation has been carried out for a speed of $120 \mathrm{~km} / \mathrm{h}$, reflecting the necessity of an emergency response to the emerging obstacle. The simulation has been carried out in MSC Adams/Car in two configurations:

- vehicle laden with a driver and a passenger having uneven masses, plus a baggage, moving on a flat and dry road surface;

- vehicle laden with a driver and a passenger having uneven masses, plus a baggage, however moving on an icy road surface with randomly occurring irregularities.

During this maneuver, the vehicle covered a distance of approximately $580 \mathrm{~m}$ measured in a straight line. The two resulting trajectories for the described case are shown in Fig. 7.

In the presented case, the area $\omega$ has been assumed to be a specific width of the road lane, in which the vehicle should remain at the beginning of the maneuver. It is therefore necessary to take into account the overall width of the vehicle applied to the width of the lane. It has been assumed that, in this case, the single lane is about $3 \mathrm{~m}$ wide. 


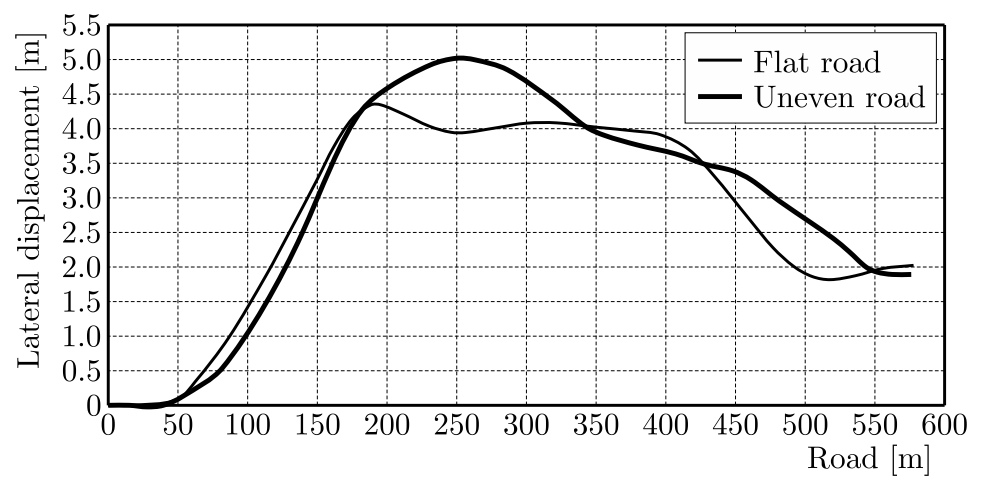

Fig. 7. Lateral motion of the vehicle in relation to the covered distance for the laden vehicle body and both road surface conditions. Source: own research

The area of feasible solutions $\Omega$, containing a part of the trajectory associated with the road length on which the featured maneuver occurs, should be referred to the total width of the road (two lanes). As mentioned previously, the resulting trajectories show the motion of the center of mass of the analysed vehicle. At each point of the trajectories, especially at their most lateral displacements, the entire width of the vehicle should be considered. It has been assumed that the whole width of the road is $6 \mathrm{~m}$, referring to the single-lane, two-way road. The total width of the simulated vehicle model is $1.9 \mathrm{~m}$. As it can be noticed, the trajectory of the vehicle motion on a flat road surface remains in the area of stability. Meanwhile, the trajectory obtained for the more extreme road conditions exits this area.

In Fig. 8, the conditions under which the vehicle remains in a particular lane of the road are schematically illustrated. Let $L$ be the total width, where the vehicle can remain during the maneuver, and $L_{1}$ - the width allowable for a representative point (here, the center of mass) of the vehicle.

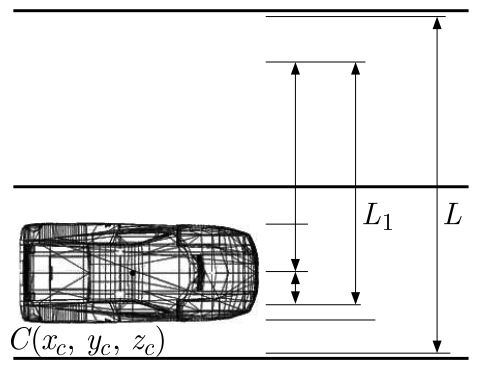

Fig. 8. The conditions of remaining in the specific width of the road, which can be assumed as stable. Source: own research

If the width of the lane is assumed as above, then at a given vehicle width (1.9 m, Fig. 2) the trajectory of the center of mass, as a representative point, may remain in the lane $L_{1}$ (about $4 \mathrm{~m}$ wide) for stable motion. However, in both cases, the trajectory extends a $4 \mathrm{~m}$ width. The conclusion is that the analysed model, for the assumed conditions on a dry and flat road surface, is at the limit of stability and exceeds it for the icy and uneven road surface.

The adoption of the above areas (width of the road) is crucial for the stability examination, namely to find the probability of the solution remaining in a specific area, which is perceived to be stable. It also seems important to define the parameters of the road for which the stability is examined.

In order to examine the stochastic technical stability of the analysed vehicle model during the maneuver, a certain part of the trajectories ranging from 170 to $420 \mathrm{~m}$ of the road has been selected, where the hypothetical obstacle avoidance occurs. This selected part is divided into 
15 classes $\left[K_{1} ; K_{15}\right]$ every $0.1 \mathrm{~m}$ (Fig. 9) in terms of lateral displacement. On the axis of the lateral displacement, the classes belonging to the $\Omega$ area are marked.

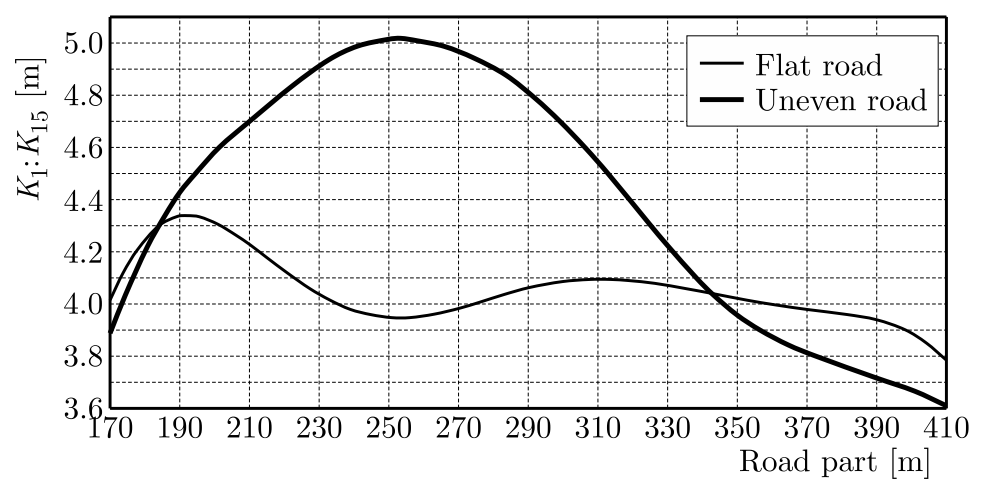

Fig. 9. The selected part of trajectories obtained for the obstacle avoidance with the division of $\Omega$ into disjoint classes $\left[K_{1} ; K_{15}\right]$. Source: own research

The frequencies of events for the obstacle avoidance [170 m; $410 \mathrm{~m}]$ have been calculated for every $20 \mathrm{~m}$ step of the road, which gave 12 subintervals.

Implementation of the disturbances took place in the domains of the road, height of road irregularities and their wavelengths. After transformation by velocity, the time, amplitude and frequency domains have been obtained. The frequencies of events have been calculated, where the solution remains in a certain class of $\Omega$ (acceptable width of the lane). Equation (4.1) has been used, however with replacing the time with the road length as the area of specificity of the considered system

$$
W\left(K_{j}\right)=\frac{T_{K j}}{T}=\frac{T_{K j} V}{T V}=\frac{S_{K j}}{S}=\frac{N_{K j} \Delta s}{N \Delta s}=\frac{N_{K j}}{N}
$$

where: $T_{K j}$ is the time when the model remains in the given class; $T$ - total time necessary to complete the considered part of the road; $S_{K j}$ - road length in the given intervals for which the model remains in the given class; $S$ - total distance of the discussed part of the road; $N_{K j}-$ number of sub-intervals for which the solution remains in the given class; $N$ - number of all sub-intervals.

Research on the stochastic technical stability of a motor vehicle model has been made by comparing the trajectories of motion and the frequencies of the solution remaining in every class of the area $\Omega$. Attention has been paid to deviation of the trajectory from the nominal position. The nominal position has been assumed for the trajectory of the vehicle moving on a flat and dry road surface (curve indicated by a thin line).

For the area of feasible solutions, two lanes of $6 \mathrm{~m}$ wide road have been assumed. The minimum width of the lane in accordance with the regulation of the Minister of Transport and Maritime Economy from 2 March 1999 is $2.5 \mathrm{~m}$. Therefore, in accordance with Fig. 9, it has been assumed that the stable motion occurs when the center of mass of the vehicle model of a certain width does not exceed a predetermined distance from the middle line dividing the road into two lanes (half width of $L_{1}$ ).

It has also been assumed that the car avoiding the obstacle at a speed of $100 \mathrm{~km} / \mathrm{h}$ should fit in the width of about $4 \mathrm{~m}$ of the $6 \mathrm{~m}$ wide road. It results from the study of the vehicle center of mass trajectory, so the width of the vehicle should be taken into account. When the motion is disturbed, i.e. when the center of mass of the car model exceeds the accepted range of a $4 \mathrm{~m}$ wide road part (icy surface), the outer wheels are dangerously close to the edge of the road or may remain in contact with the shoulder. 
The maximum amplitude values of the trajectory of the vehicle with the disturbed center of mass, for a dry road surface, are near the border of stability. For the icy road, the trajectory trespasses the accepted area of stability.

In Tables 1 and 2, the frequencies of events are presented on both the flat and dry as well as uneven and icy road surfaces. Significant differences are found in classes 3, 4, 5 and 14.

Table 1. Frequencies of events for the solution remaining in the given class of the area $\Omega$. Analysis for the flat road surface. Source: own research

\begin{tabular}{|c|c|c|c|c|c|c|c|c|c|c|c|c|c|c|c|}
\hline Class & 1 & 2 & 3 & 4 & 5 & 6 & 7 & 8 & 9 & 10 & 11 & 12 & 13 & 14 & 15 \\
\hline$N_{K j}$ & 0 & 1 & 0 & 4 & 6 & 0 & 1 & 1 & 0 & 0 & 0 & 0 & 0 & 0 & 0 \\
\hline$W\left(K_{j}\right)$ & 0 & 0.077 & 0 & 0.308 & 0.462 & 0 & 0.077 & 0.077 & 0 & 0 & 0 & 0 & 0 & 0 & 0 \\
\hline
\end{tabular}

Table 2. Frequencies of events for the solution remaining in the given class of the area $\Omega$. Analysis for the uneven road surface. Source: own research

\begin{tabular}{|c|c|c|c|c|c|c|c|c|c|c|c|c|c|c|c|}
\hline Class & 1 & 2 & 3 & 4 & 5 & 6 & 7 & 8 & 9 & 10 & 11 & 12 & 13 & 14 & 15 \\
\hline$N_{K j}$ & 1 & 1 & 2 & 1 & 0 & 0 & 1 & 0 & 1 & 1 & 0 & 1 & 1 & 2 & 1 \\
\hline$W\left(K_{j}\right)$ & 0.077 & 0.077 & 0.154 & 0.077 & 0 & 0 & 0.077 & 0 & 0.077 & 0.077 & 0 & 0.077 & 0.077 & 0.154 & 0.077 \\
\hline
\end{tabular}

In order to relate the results to the definition of stochastic technical stability, the probability of a solution remaining in a certain class of the feasible solutions area $\Omega$ should be determined first, and then the parameter $\varepsilon$ defined by formula (3.4) related to it.

It has been assumed that the probability of the solution remaining in the certain class is closely related to the frequencies determined in Tables 1 and 2. In both tables, the total frequency of event rate is equal to 1 , thus using the formula

$$
W\left(K_{j}\right)=\frac{N_{K j}}{\sum N_{K j}}
$$

where: $W\left(K_{j}\right)$ is the frequency of the solution remaining in the $j$-th class; $N_{K j}$ - number of events in the $j$-th class; $\sum N_{K j}$ - total number of events in all classes, it can be concluded that the frequency can be used to determine the probability.

Then, if the width of $\Omega$ is divided, e.g., into 15 classes and treated as an area in which the trajectory as a whole remains with the probability equal to 1 , then using to the parameter $\varepsilon$ the width of feasible solutions can be narrowed to such, that only the trajectory of the stable vehicle motion should fit without changing the area $\Omega$ but only adjusting the stable area for specific requirements. The parameter $\varepsilon$ should be adopted by subtracting as many classes from the width of the road $(\Omega)$ as under the above accepted principles correspond to the stable area.

For the presented case, the parameter $\varepsilon$ should adopt a value corresponding to the eight lower classes $\left[K_{1} ; K_{8}\right]$ occupied by the trajectory of motion obtained for the dry and flat road surface, i.e. $\varepsilon=8 / 15=0.53$, and the probability according to formula (3.4) $P=1-\varepsilon=(15-8) / 15=$ 0.47 , respectively.

As a consequence of the presented considerations, the probability of the whole trajectory remaining in the stable area should, in the presented case, be greater than or equal to 0.47 , which, for the trajectory of the vehicle on a dry and flat road, is fulfilled. For a trajectory that would run closer to the axis of the road, this probability would likely be significantly greater than 0.47. For the trajectory of the vehicle on an uneven and icy road, the probability of remaining in the stable area (substracting 14 classes) is close to zero. 


\section{Conclusions}

Based on the simulation of the vehicle motion in Adams/Car, trajectories have been obtained in two different road conditions. They show that a seemingly slight disturbance of the center of mass in the vehicle can have a greater impact on the stochastic technical stability of the vehicle motion, the greater the bad traffic conditions are.

In addition, for vehicles after an accident repair, it seems that on the basis of stability examination it would be possible to apply such a vehicle for admission to further exploitation.

Also, it has been shown how to use the definition of stochastic technical stability of mathematical models in examination of the technical objects in different conditions. The versatility of this method can provide testing in different environments (not just in the case of road vehicles). The greatest advantage of the presented method is the possibility of making an analysis based on the obtained trajectory and a comparison with the stability of real objects (example for motor vehicles presented in Zalewski (2011)).

The scope of further research can cover the stochastic technical stability analysis for different mathematical models in different road conditions and for different maneuvers.

\section{References}

1. Bogusz W., 1972, Technical Stability (in Polish), Warszawa 1972, PWN

2. Börner M., IsERmann R., 2005, The characteristic velocity stability indicator for passenger cars, Vehicle System Dynamics, $\mathbf{4 3}, 8$

3. GilLESPIE T.D., 1992, Fundamentals of vehicle dynamics, SAE International

4. Heinzl P., Lugner P., Plöchl M., 2002, Stability control of a passenger car by combined additional steering and unilateral braking, Supplement to Vehicle System Dynamics, $\mathbf{3 7}$

5. Kaneko T., Kageyama I., Tsunashima H., 2002, Braking stability of articulated vehicles on highway, Supplement to Vehicle System Dynamics, $\mathbf{3 7}$

6. Kisilowski J., Choromański W., Łopata H., 1985, Investigation of technical stochastic stability of lateral vibrations of mathematical model of rail vehicle, Engineering Transactions, $\mathbf{3 3}$

7. Kisilowski J., Kardas-Cinal E., 1993, On a certain method of examining stability of mathematical models of railway vehicles with disturbances occurring in teal objects, VSD Suplement to vol. 23, Proceedings of 13th IAVSD Symposium Held in Chendu, Sichuan, P.R. China, August 23-27

8. Kisilowski J., Zalewski J., 2008a, Chosen problems of examination of car stability, Archives of Transport Systems Telematics, 1, 1

9. KisilowsKi J., ZALEWSKI J., 2008b, On a certain possibility of practical application of stochastic technical stability, Maintenance and Reliability, $\mathbf{3 7}, 1$

10. Kuratowski K., 2012, Differential and Integral Calculus: Functions of One Variable (in Polish), PWN, Warsaw

11. Lenasi J., Danon G., ŽEŽEliJ S., 1998, Lateral stability of a braking vehicle on the friction limit, Supplement to Vehicle System Dynamics, 29

12. Mokhiamar O., ABe M., 2002, Combined lateral force and yaw moment control to maximize stability as well as vehicle responsiveness during evasive manouvering for active handling safety, Supplement to Vehicle System Dynamics, $\mathbf{3 7}$

13. MúčKA P., 2009, Sensitivity of road unevenness indicators to road waviness, Journal of Testing and Evaluation (JTE), February 
14. NabagŁo T., 2006, Synthesis of semi-active suspension control system with magneto-rheological elements (in Polish), Doctoral Thesis, Cracow University of Technology, Kraków, Poland

15. NG L., 2008, Reinforcement learning of dynamic collaborative driving, Doctoral thesis, Waterloo, Ontario, Canada

16. Using Adams, MSC Software Corporation

17. ZALEWSKI J., 2011, Modelling of the influence of car body disturbances on the stability of motion of road vehicle (in Polish), Doctoral Thesis, Warsaw University of Technology, Warszawa, Poland

Manuscript received March 16, 2015; accepted for print February 10, 2016 